\title{
Generation and sustenance of electric fields in sandstorms
}

\author{
Mustafa Mutiur Rahman $\odot,{ }^{*}$ Wan Cheng, ${ }^{\dagger}$ and Ravi Samtaney $\odot$ \\ Division of Physical Science \& Engineering, King Abdullah University of Science \& Technology, Thuwal 23955-6900, Saudi Arabia
}

(Received 3 February 2020; accepted 28 December 2020; published 20 January 2021)

\begin{abstract}
Sandstorms are frequently accompanied by intense electric fields and lightning. In a very narrow region close to the ground, sand particles undergo a charge exchange during which larger-sized sand grains become positively charged and smaller-sized sand grains become negatively charged, and then all particles become suspended by the turbulent fluid motion. Although the association of intense electric fields with sandstorms has long been observed, the mechanism that causes these intense electric fields has not yet been described. Here, we hypothesize that differently sized sand particles are differentially transported by turbulence in the flow, resulting in a large-scale charge separation and a consequential large-scale electric field. To confirm our hypothesis, we combined a large-eddy simulation framework comprising a turbulent atmospheric boundary layer and movement of sand particles with an electrostatic Gauss law to investigate the physics of the electric fields in sandstorms. We varied the strength of the sandstorm from weak to strong as parametrized by the number density of the entrained sand particles. Our simulations reproduced observational measurements of both mean and root mean squared fluctuation values of the electric field. Our results allowed us to propose a law in which the electric field scales to two-thirds of the power of the concentration of the sand particles in weak to medium strength sandstorms.
\end{abstract}

DOI: 10.1103/PhysRevResearch.3.L012008

\section{INTRODUCTION}

As far back as 1850, Michael Faraday noted that electric fields accompanied sandstorms:

"I received your letter respecting Dust Storms...The quantity of Electricity which you obtain at the time is enormous...That it [electricity] accompanies them [dust storms], there is no doubt of, but then, that may be as much in the way of effect as cause" [1].

In this letter, Faraday noted the misconception that electricity caused sandstorms. Since then, the idea that intense electric fields are generated during sandstorms has been widely accepted.

Zhang et al. [2] reported a maximum average intensity of electric fields of about $200 \mathrm{kV} / \mathrm{m}$ with instantaneous values exceeding $2.5 \mathrm{MV} / \mathrm{m}$ in a sandstorm. Such intense electric fields can cause wildfires, communication disruptions, and explosions [3,4]. Even on Mars, sandstorms cause problems for rovers and satellites because of the electric fields that are generated [5].

\section{A. Turbulence and large-scale charge separation}

A sandstorm is a complex meteorological event that generally involves a storm front, turbulent flow with a high

\footnotetext{
*Mustafa.Rahman@kaust.edu.sa

${ }^{\dagger}$ Also affiliated with Graduate Aerospace Laboratories, California Institute of Technology, CA 91125, USA.

Published by the American Physical Society under the terms of the Creative Commons Attribution 4.0 International license. Further distribution of this work must maintain attribution to the author(s) and the published article's title, journal citation, and DOI.
}

Reynolds number, the transport of sand, and an accompanying electric field.

Although sandstorms have been the subject of intensive research [6-9], a satisfactory explanation for why large-scale electric fields occur during sandstorms has not yet been offered in the literature. Recent theoretical work and small-scale laboratory experiments [10] have produced simple predictive models for the charging of granular materials in collisional flows. However, these small-scale laboratory experiments (conducted in the presence of an external electric field) cannot predict or explain why large-scale charge separation and self-sustaining electric fields occur during sandstorms. Furthermore, as discussed below, the main body of a sandstorm experiences negligible interparticle collisions. To produce a flash of lightning, the electric field must exceed the dielectric strength $(3 \mathrm{MV} / \mathrm{m})$ [11]. Electric fields of such intensity cannot arise from triboelectrification or other charge-generating mechanisms, as previously suggested [11].

Rather, to understand the electric field mechanism during sandstorms, we must consider another physical phenomenon-turbulence. Here, we hypothesize that turbulent transport of differently sized sand particles in a sandstorm leads to charge separation and generation of intense electric fields. Turbulence also sustains the electric fields during the storm.

To confirm our hypothesis, we developed a simulation framework to solve the equations governing turbulent atmospheric flow along with the transport of charged sand particles to estimate the intensity of the electric fields.

Direct numerical simulations (DNSs) [12,13] are limited to relatively low Reynolds numbers and cannot reproduce realistic conditions of a sandstorm. A DNS of homogeneous isotropic turbulence in a cubic domain with periodic boundary 
conditions (BCs) [13] implies only a zero mean electric field. Moreover, these DNS investigations have not been compared with actual measurements of electric fields in sandstorms.

Our main goal was to model the electric fields in highReynolds-number atmospheric flows. This goal allowed us to exclude sandstorm fronts from the framework because our modeling of the atmospheric turbulent boundary flow in a statistically steady state captured conditions away from the sandstorm front.

\section{B. Physical characteristics of sandstorms and modeling approach}

Sandstorms are turbulent air motions that contain suspended solid sand particles. During a sandstorm, sand particles are entrained by large-scale swirling motions in the turbulent atmospheric boundary layer with a characteristic height (denoted by $\delta$ ) of $O(100 \mathrm{~m})$. The large-scale integral length of a sandstorm is of the order of $O(\mathrm{~km})$. Thus, a typical atmospheric boundary-layer Reynolds number $\left(\operatorname{Re}_{\delta}\right)$ in a sandstorm can exceed $10^{9}$. Resolving all these turbulent scales with a DNS technique is not practically viable; we therefore resort to computing the fluid turbulence with the large-eddy simulation (LES) approach. Wind tunnel experiments have shown that sand particles smaller than $250 \mu \mathrm{m}$ acquire a negative charge, whereas particles larger than $500 \mu \mathrm{m}$ acquire a positive charge [14].

These observations were corroborated by other experiments $[10,15]$. Most numerical simulations related to sandstorms focus on the saltation and creeping modes [16] but not on the suspension mode, in which sand particles are transported by the airflow and do not settle back to the ground. Here, we consider a sandstorm as a mixture of solid sand particles in the suspension mode in the atmospheric boundary layer at a statistically steady state. We choose the suspension mode and not the other modes where collisions between sand particles mostly occur near the ground in an extremely thin layer $[O(10-100 \mathrm{~mm})]$ where the volume fraction of sand is large and where the sand particles can exchange electrical charges [17]. The collision rate between sand particles diminishes extremely rapidly above this height. In the suspension mode, there are practically no collisions between suspended sand particles.

In addition, the suspension mode is well characterized by dilute particulate flow with no charge exchange and one-way coupling from fluid to solid.

The particle-size distribution (PSD) in this mode lies in the Eulerian and equilibrium-Eulerian range (see classification in Balachandar and Eaton [18]), and the Stokes number is small. We adopt the Eulerian description for the particulate phase of sandstorms.

\section{Governing equations}

In LES, the filtered incompressible Navier-Stokes equations describe the fluid phase of a sandstorm as follows:

$$
\begin{aligned}
\nabla \cdot \tilde{\mathbf{u}} & =0, \\
\partial_{t} \tilde{\mathbf{u}}+\tilde{\mathbf{u}} \cdot \nabla \tilde{\mathbf{u}} & =v \nabla^{2} \tilde{\mathbf{u}}-\nabla \cdot \mathbf{T}-\left(\partial_{x} p_{0} \hat{\mathbf{e}}_{x}+\nabla \tilde{p}^{\prime}\right) / \rho_{f},
\end{aligned}
$$

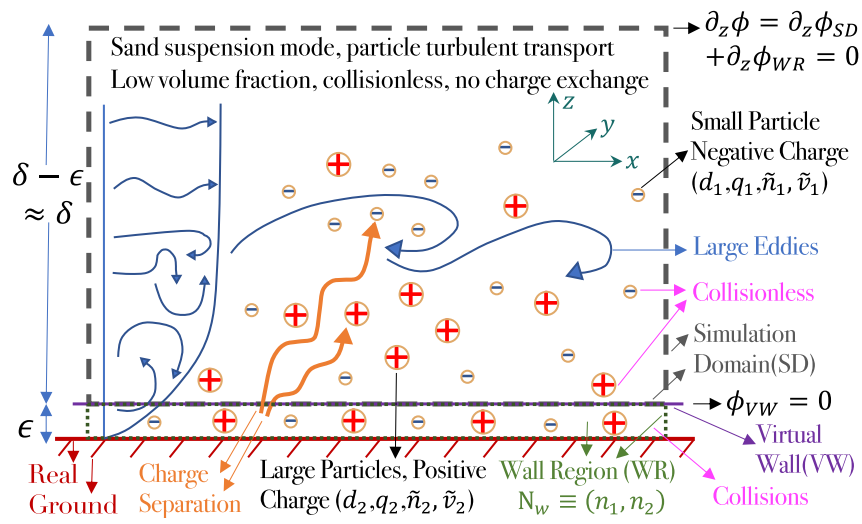

FIG. 1. The simulation domain (SD). The height of the atmospheric boundary layer $\delta$ is modeled as a turbulent half-channel flow with streamwise and spanwise periodic conditions. The lower boundary is modeled as a virtual wall (VW) at $\epsilon(\ll \delta)$ height.

where $\tilde{\mathbf{u}}$ is the filtered fluid velocity, $\partial_{x} p_{0} \hat{\mathbf{e}}_{x}$ is a source term corresponding to a fixed streamwise pressure gradient (necessary to maintain the flow because the atmospheric boundary layer is modeled as a half channel), $\tilde{p}^{\prime}$ is the perturbed pressure, $v$ is the kinematic viscosity, and $\mathbf{T}=\widetilde{\mathbf{u u}}-\tilde{\mathbf{u}} \tilde{\mathbf{u}}$ is the subgrid stress (SGS) tensor. We coupled the fluid equations with equations for the conservation of mass and momentum for charged solid sand particles. We determined that a oneway coupling between the fluid and the solid phases was reasonable by considering the low volume fraction of the sand phase $\left(<10^{-6}\right)[18]$. The mass and momentum conservation for the solid phase follows from the direct quadrature method of moments (DQMOM) [19] in which the weights and abscissas of the quadrature method rather than the moments of the PSD are directly tracked.

$$
\begin{aligned}
& \partial_{t} \tilde{n}_{s}+\nabla \cdot\left(\tilde{n}_{s} \tilde{\mathbf{v}}_{s}\right)=0, \\
& m_{s} \partial_{t} \tilde{\mathbf{v}}_{s}+m_{s} \tilde{\mathbf{v}}_{s} \cdot \nabla \tilde{\mathbf{v}}_{s}=\tilde{\mathbf{D}}_{s}+q_{s} \mathbf{E}, \\
& \tilde{\mathbf{D}}_{s}=3 \pi v \rho_{f} d_{s} \cdot\left(1+0.15 \operatorname{Re}_{s}^{0.687}\right) \cdot\left(\tilde{\mathbf{u}}-\tilde{\mathbf{v}}_{s}\right), \\
& \mathbf{E}=-\nabla \phi, \quad \varepsilon \nabla^{2} \phi=-\Sigma, \quad \Sigma=\sum_{s=1}^{s=\mathcal{S}} \tilde{n}_{s} q_{s} .
\end{aligned}
$$

We sampled the distribution function for the sand particles at $\mathcal{S}$ points. Here, $\mathcal{S}(=2)$ is thus the total number of species considered. Also, $\tilde{\mathbf{v}}_{s}, \mathbf{D}_{s}$, and $q_{s} \mathbf{E}$ are the velocity, the correlation of drag force $[18,19]$, and the electrostatic force on species $s$, respectively. Furthermore, $\operatorname{Re}_{s}=\left|\tilde{\mathbf{u}}-\tilde{\mathbf{v}}_{s}\right| d_{s} / \nu$ is the Reynolds number of the particle. The electrostatic potential is denoted by $\phi, \varepsilon$ is the permittivity of the atmosphere, and $\Sigma$ is the local net charge density.

\section{Computational setup and BCs}

We solved these coupled equations for charged sand particles suspended in the atmospheric boundary layer, which we modeled as a turbulent half-channel flow [20]. The physical setup and computational domain are depicted in Fig. 1. In our setup, the lower boundary is not at ground level but at an elevated height (denoted as $\epsilon$ ) extending into the log layer of the channel flow. This setup is akin to the wall-modeled LES 
TABLE I. Cases and parameters in the simulations. All length scales are normalized to the viscous length scale, i.e., $z^{+}=z u_{\tau} / v$, where $u_{\tau} \equiv \sqrt{\tau_{w} / \rho_{f}}$ is the friction velocity and $\tau_{w}$ is the wall shear stress approximated as $\sqrt{-\partial_{x} p_{0} \delta}$. Here, $\xi^{+}$is the smallest resolved eddy in LES. Also, $\rho_{s}$ is the density of the particle species $(s)$ as $\rho_{s}=m_{s} /\left(\pi d_{s}^{3} / 6\right)$, where $d_{s}$ is its size, and $\eta$ is on the Kolmogorov length scale.

\begin{tabular}{lc||cc|cc}
\hline \hline Case & $N_{w}$ & $N_{1}=\left(n_{1}, n_{2}\right)$ & $(20,3.4) \mathrm{Mm}^{-3}$ & $R_{\delta}$ & $10^{9}$ \\
I & $N_{1}$ & Charge $\left(q_{1}, q_{2}\right)$ & $(-4,2.24) \mathrm{fC}$ & $\delta$ & $1000 \mathrm{~m}$ \\
II & $4 N_{1}$ & $\left(d_{1} / \eta, d_{2} / \eta\right)$ & $(1.12,2.81)$ & Domain & Grid \\
III & $10 N_{1}$ & $\rho_{1}=\rho_{2}$ & $2650 \mathrm{~kg} / \mathrm{m}^{3}$ & $(768,19,1) \delta$ \\
III-B & $12 N_{1}$ & $\Delta x^{+}=\Delta y^{+}$ & $4 \Delta z^{+}=16 \times 10^{5}$ & $\xi^{+}$ & $\Delta z^{+}$ \\
IV & $40 N_{1}$ & $-\partial_{x} p_{0}$ & $0.108 \mathrm{~g} /(\mathrm{ms})^{2}$ & $\epsilon^{+}$ & $7.5 \times 10^{4}$ \\
\hline \hline
\end{tabular}

approach of Chung and Pullin [21]. We imposed periodic BCs in the streamwise and spanwise directions for both the fluid and solid phases. At the virtual wall (VW), the fluid velocity is modeled with dynamic Dirichlet BC [21].

We assumed that the sand particles undergo collisions and exchange charges below the VW, and then they become entrained into the flow. The physics of such a charge exchange and liftoff process is complex to model. One approach is to parametrize this phenomenon as a flux BC of charged sand particles into the flow, which is proportional to the number density of sand species and dependent on many factors, such as soil humidity and ground temperature, as well as other wind erosion factors. In other words, we cannot consider just the Reynolds number of the atmospheric flow. A simpler alternative approach is to specify a Dirichlet BC for the particle number density distribution $\left(N_{w}\right)$ at the VW. In this case, we specified the $\mathcal{S}$ vector of the number density values at the VW, which parameterizes the strength of the sandstorm. For the electrostatic potential, we used a zero wall-normal gradient (Neumann BC) at the top $\left(\partial_{z} \phi_{\mathrm{SD}}=0\right)$, along with the Dirichlet $\mathrm{BC}\left(\phi_{\mathrm{VW}}=0\right)$ at the VW. To maintain charge neutrality in the total domain [the simulation domain (SD) plus the wall region (WR)], we equally (and uniformly) distributed the excess charge in the SD but with an opposite sign in the region below the VW.

\section{NUMERICAL METHODOLOGY}

Following previous work [22], we employed the stretched spiral-vortex SGS model coupled with a wall model that uses an inner-scaling ansatz to derive an ordinary differential equation for the VW velocity for this LES of incompressible turbulent flows [Eq. (1)]. The numerical solver utilized a fractional-step method with an energy-conserving fourthorder finite-difference scheme on a staggered mesh [23]. We computed the dispersed solid phase [Eqs. (2) and (3)] by using the Eulerian approach. We then modeled the electrical interactions between charged particles with respect to Gauss's law [Eq. (4)]. We used a multigrid technique to solve the Poisson equation governing the electrostatic potential by taking into account the charge distribution below the VW. We extensively tested and validated the numerical code $[23,24]$.

\section{A. Simulations}

We sampled the particle distribution function of the number density at two points $(\mathcal{S}=2)$, which means that the sand phase comprised two sizes. The representative (ideal) electric charge of the species of each size was $Q_{1} \equiv\left(q_{1}, q_{2}\right)$. All parameters are presented in Table I. We selected the typical concentration at the bottom of the suspension [top of saltation, $O(1 \mathrm{~m})]$ to be $N_{1}=\left(n_{1}, n_{2}\right)$ [25].

We varied $N_{w}$ from $N_{1}$ to $40 N_{1}$ and labeled it as follows: I ("weak"), II ("moderate"), III ("strong"), and IV ("very strong"). We included Case III-B (close to Case III) because it corresponds to field measurements of strong sandstorms. We note that we performed simulations of the fluid flow in the laminar regime comprising a Poiseuille velocity profile. The laminar solution resulted in zero mean and root mean square (RMS) of fluctuations of the electric field values, further lending credence to our hypothesis.

\section{RESULTS}

\section{A. Instantaneous electric fields}

We plot the time variation of $E_{z}$ in Fig. 2 upon which we superimpose field observations $[2,26]$.

We emphasize that the turbulent flow field is in a statistically steady state and that the time origin is therefore somewhat arbitrary. We align the minimum of $E_{z}$ [in Fig. 2(a)] and align the signal variation of $E_{z}$ [in Fig. 2(b)] between the observations and simulations.

We remark that this favorable instantaneous comparison between our simulation results and the field measurements is somewhat serendipitous. It stems from an extensive querying of our simulation data in space and time. We extract the electric field from the simulation data at various spatial (streamwise and spanwise) positions to compare and match its temporal variation with the in situ sandstorm measurements. Since the exact conditions of any particular sandstorm are virtually impossible to reproduce, the match between the instantaneous observations and simulations is fortuitous. It is evidently more important to match the mean and fluctuations of the electric field (as discussed below). Nonetheless, the overall average magnitude of $E_{z}$ from our simulations compares well with the field observations, although the simulations also depict higher frequencies in the variation of $E_{z}$ than the observations (which may be limited due to instrumentation). These results demonstrate the validity of our code and also show the robustness of the simulation framework and numerical methods.

\section{B. Varying sandstorm strength}

Cases I-IV signify sandstorms that increase in strength progressively from weak to very strong. We compute the 

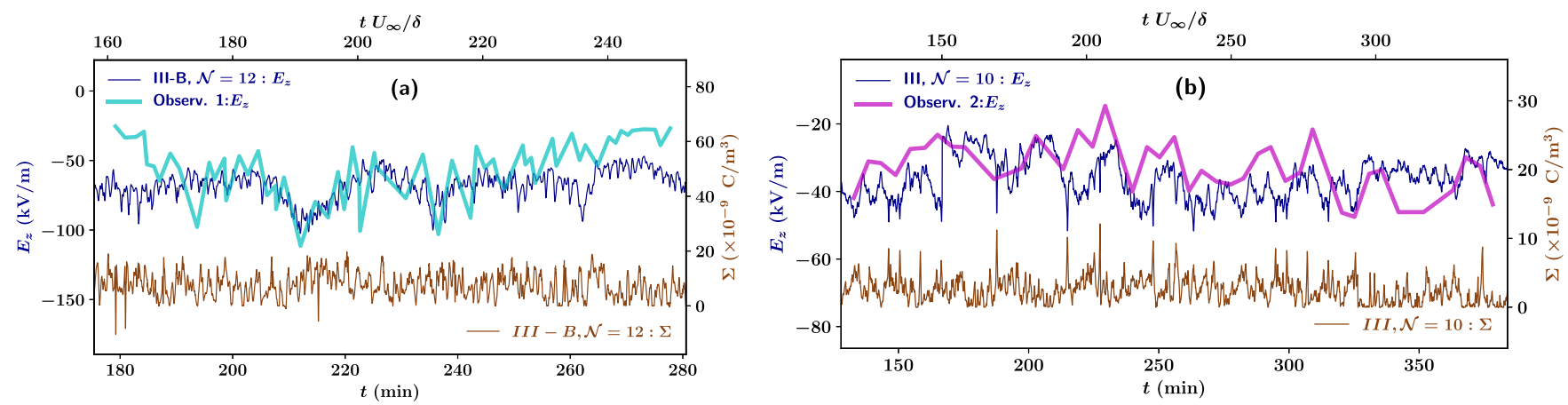

FIG. 2. Time history of the simulated instantaneous wall-normal electric field $\left(E_{z}\right)$ at the midspan, midstream grid location (blue) along with the charge density variation (brown) at (a) $14.2 \mathrm{~m}$, Case III-B, and (b) $35 \mathrm{~m}$, Case III, where $\mathcal{N}=N_{w} / N_{1}$. Superimposed on the simulations are in situ measurements by Zhang et al. [2] (Observ. 1) and Zhang et al. [26] (Observ. 2).

flow until it is statistically steady, after which the vertical component of the electric field $E_{z}$ is time averaged over the horizontal plane $\bar{E}_{z}$ and the RMS of $E_{z}^{\prime}$. We plot the variation in altitude of $\bar{E}_{z}$ and $E_{z}^{\prime}$ and the mean charge density $\bar{\Sigma}$ for all four cases in Fig. 3. The magnitude of the mean wall-normal electric field of each case in Fig. 3(a) decreases with height because of the decrease in the mean profile of the charge density. As the strength of the sandstorm increases, the mean and fluctuation magnitudes of the electric field also increase. For each case, the magnitudes of the mean and RMS values decrease with altitude, as observed in Figs. 3(a) and 3(b). The mean magnitudes of the horizontal components of the electric field are negligible because of the streamwise and spanwise periodic BC, but the RMS values are of the same order of magnitude as the vertical component.

In Case III, we note the instantaneous maximum $|\mathbf{E}|=200 \mathrm{kV} / \mathrm{m}$ and range of $E_{z} \in(-70,30) \mathrm{kV} / \mathrm{m}$ in the domain. In cases III, III-B, and IV, the maximum magnitude of the horizontal electric field exceeds $100 \mathrm{kV} / \mathrm{m}$, which has also been observed in the field [5]. In the weak sandstorm case (Case I), the near-wall average electric field $\left(\bar{E}_{z}\right)$ is close to the observed electric field $\left(\bar{E}_{z} \approx-10 \mathrm{kV} / \mathrm{m}\right)$ [26], whereas in the moderate sandstorm case (Case II), $\bar{E}_{z} \approx-30 \mathrm{kV} / \mathrm{m}$, and in the strong sandstorm case (Case III), $\bar{E}_{z} \approx-80 \mathrm{kV} / \mathrm{m}$, which agree with field measurements [2,26]. The RMS fluctuation of the near-wall vertical electric field in Case II [Fig. 3(b)] is close to $7 \mathrm{kV} / \mathrm{m}$ as observed in the field [27]. The instantaneous vertical electric field may be in the same or opposite direction as the Earth's background electric field. The instantaneous fluctuations at some locations are sufficiently high to cause a reversal in the direction of the vertical electric field component. Such a change in the direction of the electric field has been observed in the field [26] and similarly observed in the saltation layer [28]. The mean charge densities plotted in Fig. 3(c) show that their magnitudes are comparable to the measurements in the field [29] and at their maximums close to the ground. The electrostatic forces on the particles are such that they tend to reduce the charge separation overall, although the high-intensity turbulent fluid fluctuations counterbalance this drive.

The mean and RMS net electric field, as shown in Figs. 4(a) and 4(b), decrease with height, and both are directly correlated with the decrease in the mean and RMS profile of the charge density. The vertical electric field fluctuations, as shown in Fig. 3(b), are at their maximums close to the ground because of the high turbulent intensity, which is at its maximum there. We note that the largest charge density fluctuation also occurs in the vicinity of the wall and is well correlated with the turbulent intensity of the flow.

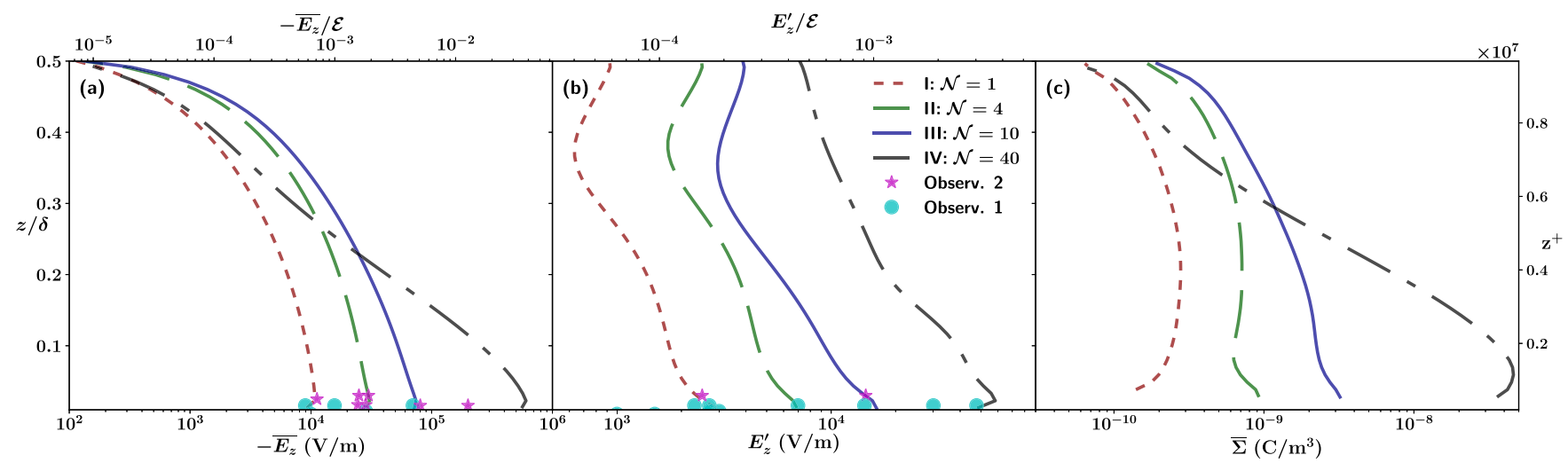

FIG. 3. Altitude variation of the wall-normal electric field (a) mean (time and streamwise/spanwise averaged) of $\bar{E}_{z}$, (b) root mean square (RMS) of fluctuations $E_{z}^{\prime}$, and (c) mean charge density, where $\mathcal{E}=\delta \Xi / \varepsilon$ and $\Xi=\mathcal{S} \mathrm{M}\left(n_{1}\left|q_{1}\right|, n_{2}\left|q_{2}\right|\right)$ are, respectively, the reference electric field value and the reference charge density value corresponding to Case I $[M(a, b)=$ mean of $a$ and $b]$. The symbols are mean and RMS values synthesized from various sandstorm measurements by Zhang et al. [2] (circles) and Zhang et al. [26] (stars). 


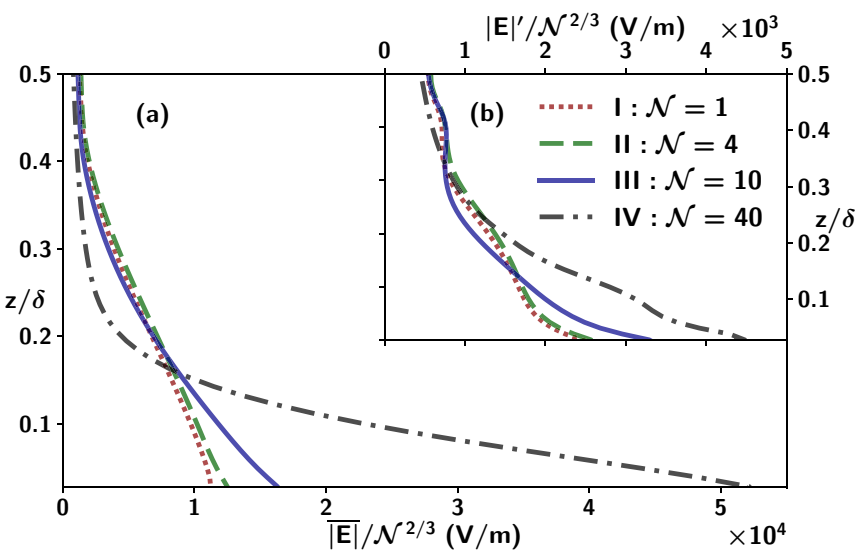

FIG. 4. Altitude variation of the scaled net electric field magnitudes of (a) mean $\overline{|\mathbf{E}|}$, and (b) RMS fluctuations $|\mathbf{E}|^{\prime}$, both scaled by $\mathcal{N}^{\frac{2}{3}}$

\section{Self-similarity}

In all simulations considered here, the fluid turbulence is identical because of the one-way coupling between the fluid and solid phases. The differences in the charge density stem from the differences in the flux of the sand particles into the turbulent flow. The fundamental electrostatic charge interaction scales as $E \propto q / r^{2}$. Combined with the fact that, in dilute particle flow, the average interparticle distance $\left(\propto \tilde{n}_{s}^{-1 / 3}\right)$ for a low volume fraction of particles leads to the $2 / 3$ scaling power law for the electric field, i.e., $E \propto 1 / r^{2} \propto \mathcal{N}^{2 / 3}$.

The mean and RMS scaled electric field magnitude (normalized by $\mathcal{N}^{2 / 3}$ ) as a function of normalized distance are plotted in Fig. 4. The scaling $E \propto \mathcal{N}^{2 / 3}$ agrees well with the simulations for the dilute particle flow, cases I and II, because the average interparticle relation is well satisfied.

\section{SUMMARY}

We presented a framework to simulate a sandstorm modeled as the flow of charged sand particles in a turbulent flow of a statistically steady atmospheric boundary layer. The physics of the electric field generation is a complex nonlinear process involving turbulent flow interactions with suspended solid charged particles; the solid particle phases interact via Coulombic electrostatic interactions.

The dynamics of the phenomenon are that the charged particles become entrained by the turbulent flow field; the turbulent flow transports the smaller sized (lighter) sand particles differently than it transports the larger (heavier) ones. Owing to their larger inertia, the larger particles do not follow the fluid flow as closely as the lighter ones. The turbulent flow causes fluctuations in the number density and spatial variation between the lighter and heavier charged species. The charge separation, in turn, creates the electrical field. A further increase in the electrical charge concentration of the particles can reach the breakdown field in air, which may trigger lightning (not modeled here). Our computed mean and RMS values match those observed in sandstorms.

Our analysis demonstrates that the turbulent transport of charged sand particles is crucially responsible for the observed electric fields in sandstorms. The electric fields produced under sandstorm conditions may be in the same or opposite direction to Earth's normal electric field and decrease with altitude. We propose a simple scaling of $\overline{|\mathbf{E}|},|\mathbf{E}|^{\prime} \propto \mathcal{N}^{2 / 3}$, which holds for weak to moderate strength sandstorms. We posit that the level and frequency of occurrence of atmospheric turbulence will increase [30] in the coming decades with the impact of climate change, making studies such as this relevant to everyday weather events. Even though this paper concentrates on earthbound dust suspensions, our simulation framework may also be useful for modeling severe Martian sandstorms.

\section{ACKNOWLEDGMENTS}

This paper was supported by KAUST under Awards No. URF/1/1704-01-01 and No. BAS/1/1/1349-01-1. Cray $\mathrm{XC} 40$ - Shaheen-II was used for simulations.
[1] P. F. H. Baddeley, Whirlwinds and Dust-Storms of India (Bell \& Daldy, London, 1860).

[2] H. F. Zhang, T. Wang, J. J. Qu, and M. H. Yan, An experimental and observational study on the electric effect of sandstorms, Chinese J. Geophys. 47, 53 (2004).

[3] B. A. Keith, A suggested classification of great plains dust storms, Trans. Kansas Acad. Sci. (1903-) 47, 95 (1944).

[4] H. F. Eden and B. Vonnegut, Electrical breakdown caused by dust motion in low-pressure atmospheres: Considerations for Mars, Science 180, 962 (1973).

[5] T. L. Jackson and W. M. Farrell, Electrostatic fields in dust devils: An analog to Mars, IEEE Trans. Geosci. Remote Sens. 44, 2942 (2006)

[6] Y. Shao, Physics and Modeling of Wind Erosion, Vol. 37 (Springer Science \& Business Media, Dordrecht, 2008).

[7] X. Zheng, Mechanics of Wind-Blown Sand Movements (Springer Science \& Business Media, Heidelberg, 2009).
[8] X.-J. Zheng, Electrification of wind-blown sand: Recent advances and key issues, Eur. Phys. J. E 36, 138 (2013).

[9] Q. Jianjun, M. Yan, G. Dong, H. Zhang, R. Zu, W. Tuo, A. Zhao, Z. Xiao, F. Li, and B. Yang, Wind tunnel simulation experiment and investigation on the electrification of sandstorms, Sci. China Ser. D: Earth Sci. 47, 529 (2004).

[10] T. Pähtz, H. J. Herrmann, and T. Shinbrot, Why do particle clouds generate electric charges? Nat. Phys. 6, 364 (2010).

[11] S. P. Kanagy and C. J. Mann, Electrical properties of eolian sand and silt, Earth Sci. Rev. 36, 181 (1994).

[12] H. Grosshans and M. V. Papalexandris, Direct numerical simulation of triboelectric charging in particle-laden turbulent channel flows, J. Fluid Mech. 818, 465 (2017).

[13] M. Di Renzo and J. Urzay, Aerodynamic generation of electric fields in turbulence laden with charged inertial particles, Nat. Commun. 9, 1 (2018). 
[14] X. J. Zheng, N. Huang, and Y.-H. Zhou, Laboratory measurement of electrification of wind-blown sands and simulation of its effect on sand saltation movement, J. Geophys. Res. 108, 4322 (2003).

[15] V. Lee, S. R. Waitukaitis, M. Z. Miskin, and H. M. Jaeger, Direct observation of particle interactions and clustering in charged granular streams, Nat. Phys. 11, 733 (2015).

[16] J. F. Kok and N. O. Renno, Electrostatics in Wind-Blown Sand, Phys. Rev. Lett. 100, 014501 (2008).

[17] Y. Zhang, J. Yang, D. Liu, X. Wei, and L. Yu, Sand storms: CFD analysis of Reynolds stress and collision stress of particles near sand bed, Particuology 8, 325 (2010).

[18] S. Balachandar and J. K. Eaton, Turbulent dispersed multiphase flow, Annu. Rev. Fluid Mech. 42, 111 (2010).

[19] O. Desjardins, R. O. Fox, and P. Villedieu, A quadrature-based moment method for dilute fluid-particle flows, J. Comput. Phys. 227, 2514 (2008)

[20] M. Calaf, C. Meneveau, and J. Meyers, Large eddy simulation study of fully developed wind-turbine array boundary layers, Phys. Fluids 22, 015110 (2010).

[21] D. Chung and D. I. Pullin, Large-eddy simulation and wall modeling of turbulent channel flow, J. Fluid Mech. 631, 281 (2009).

[22] M. M. Rahman, W. Cheng, R. Samtaney, and J. Urzay, Largeeddy simulations of sandstorms as charged-particle suspensions in turbulent boundary layers, in Proceedings of the 2016 Center for Turbulence Research Summer Program (Stanford University, 2016), p. 45.
[23] M. M. Rahman and R. Samtaney, Modeling and analysis of large-eddy simulations of particle-laden turbulent boundary layer flows, in AIAA SciTech Forum - 55th AIAA Aerospace Sciences Meeting (AIAA, Reston, VA, 2017), p. 0981.

[24] M. M. Rahman and R. Samtaney, Particle concentration variation for inflow profiles in high Reynolds number turbulent boundary layer, in Fluids Engineering Division Summer Meeting (American Society of Mechanical Engineers, 2020), Vol. 83723, p. V002T04A022.

[25] X. Liu and Z. Dong, Experimental investigation of the concentration profile of a blowing sand cloud, Geomorphology 60, 371 (2004).

[26] X. Zhang, D. Li, and T.-L. Bo, The variation of the vertical electric field $\left(\mathbf{E}_{\mathrm{z}}\right)$ with height during dust storms and the effects of environmental variables on $\mathbf{E}_{\mathrm{z}}$, Granular Matter 20, 33 (2018).

[27] T.-L. Bo and X.-J. Zheng, A field observational study of electrification within a dust storm in Minqin, China, Aeolian Res. 8, 39 (2013).

[28] D. S. Schmidt, R. A. Schmidt, and J. D. Dent, Electrostatic force on saltating sand, J. Geophys. Research Atmos. (1984-2012) 103, 8997 (1998).

[29] H. Zhang and X. Zheng, Quantifying the large-scale electrification equilibrium effects in dust storms using field observations at Qingtu Lake Observatory, Atmospheric Chem. Phys. 18, 17087 (2018).

[30] L. N. Storer, P. D. Williams, and M. M. Joshi, Global response of clear-air turbulence to climate change, Geophys. Res. Lett. 44, 9976 (2017). 